\title{
Controlling the Movement of Magnetic Iron Oxide Nanoparticles Intended for Targeted Delivery of Cytostatics
}

\author{
Yana Toropova' \\ Dmitry Korolev' \\ Maria Istomina ${ }^{1,2}$ \\ Galina Shulmeyster' \\ Alexey Petukhov 1,3 \\ Vladimir Mishanin' \\ Andrey Gorshkov ${ }^{4}$ \\ Ekaterina Podyacheva' \\ Kamil Gareev (D) ${ }^{2}$ \\ Alexei Bagrov ${ }^{5}$ \\ Oleg Demidov 6 ,7
}

'Almazov National Medical Research Centre, Ministry of Health of the Russian Federation, Saint-Petersburg, 19734I, Russian Federation; ${ }^{2}$ Saint Petersburg Electrotechnical University "LETI", SaintPetersburg, 197376, Russian Federation; ${ }^{3}$ Personalised Medicine Center, Almazov National Medical Research Centre, Ministry of Health of the Russian Federation, Saint-Petersburg, 19734I, Russian Federation; ${ }^{4} \mathrm{FSBI}$ "Research Institute of Influenza named after A.A. Smorodintsev " Ministry of Health of Russian Federation, Saint-Petersburg, Russian Federation; ${ }^{5}$ Sechenov Institute of Evolutionary Physiology and Biochemistry, Russian Academy of Sciences, Saint-Petersburg, Russian Federation; ' ${ }^{6}$ nstitute of Cytology RAS, Saint-Petersburg, 194064, Russian Federation; ${ }^{7}$ INSERM UI23I, Faculty of Medicine and Pharmacy, University of Bourgogne-Franche Comté, Dijon, France

Correspondence: Yana Toropova Almazov National Medical Research Centre, Ministry of Health of the Russian Federation, Saint-Petersburg, 19734I, Russian Federation

Tel +7 98I 9564800

Fax +7 8124997069

Email yana.toropova@mail.ru
Background: A promising approach to solve the problem of cytostatic toxicity is targeted drug transport using magnetic nanoparticles (MNPs).

Purpose: To use calculation to determine the optimal characteristics of the magnetic field for controlling MNPs in the body, and to evaluate the efficiency of magnetically controlled delivery of MNPs in vitro and in vivo to a tumour site in mice.

Material and Methods: For the in vitro study, reference MNPs were used, while for in vivo studies, MNPs coated in polylactide including fluorescent indocyanine (MNPs-ICG) were used. The in vivo luminescence intensity study was performed in mice with tumours, with and without of a magnetic field at the sites of interest. The studies were performed on a hydrodynamic stand developed at the Institute of Experimental Medicine of the Almazov National Medical Research Centre of the Ministry of Health of Russia.

Results: The use of neodymium magnets facilitated selective accumulation of MNPs. One minute after the administration of MNPs-ICG to mice with a tumour, MNPs-ICG predominantly accumulated in the liver, in the absence and presence of a magnetic field, which indicates its metabolic pathway. The intensity of the fluorescence in the animals' livers did not change over time, although an increase in fluorescence in the tumour was observed in the presence of a magnetic field.

Conclusion: This type of MNP, used in combination with a magnetic field of calculated strength, can form the basis for the development of magnetically controlled transport of cytostatic drugs into tumour tissue.

Keywords: fluorescence analysis, indocyanine, iron oxide nanoparticles, magnetically controlled delivery of cytostatics, tumor targeting

\section{Introduction}

Oncological diseases are one of the leading causes of death worldwide. At the same time, the dynamics of the increase in morbidity and mortality from tumor diseases remains. ${ }^{1}$ Today chemotherapy used remains one of the main treatments for different tumors. At the same time, the development of ways to reduce the systemic toxicity of cytostatics remains relevant. One of the promising approaches aimed at solving the problem of their toxicity is the method of targeted transport of drugs using nanoscale carriers that provide local accumulation of drugs in tumor tissue without increasing their concentration in healthy organs and tissues. ${ }^{2}$ This approach makes it possible to increase the efficiency and targeting of the effect of chemotherapy drugs on tumor tissue, while reducing their systemic toxicity. 
Among a wide range of nanoparticles considered for use in targeted delivery of cytostatics, magnetic nanoparticles (MNPs) are of particular interest, because of their unique chemical, biological, and magnetic characteristics, which ensure their versatility. Thus, magnetic nanoparticles can be used as heat-generating systems for hyperthermic action on a tumour (magnetic hyperthermia). It is also possible to use them as a diagnostic agent (magnetic resonance diagnostics). ${ }^{3-5}$ The use of these properties, in combination with the possibility of accumulation of MNPs in a certain area, by using an external magnetic field, to provide targeted delivery of pharmacological agents opens up the prospect of creating a multifunctional, magnetically controlled system for targeted delivery of cytostatics to the site of a tumour. Such a system would include MNPs and a magnetic field to control their movement in the body. In this case, both an external magnetic field and magnetic implants, placed in the area of the body containing the tumour, could act as the source of a magnetic field. ${ }^{6}$ The first approach has serious drawbacks, including the need to use a specialized device for magnetic targeting of drugs and the need to train personnel for the procedure. Furthermore, this approach is limited by high cost and is applicable only to "superficial" tumours, located close to the surface of the body. The alternative approach of using magnetic implants expands the range of application of this technology, facilitating it use for tumours located in different parts of the body. Both individual magnets and magnets integrated into intracavitary stents can act as implants, which are used in cases of tumour damage to hollow organs, to ensure their patency. However, based on our own unpublished research, these do not have sufficient magnetic properties to ensure retention of MNPs from the bloodstream.

The effectiveness of magnetically controlled drug delivery depends on many factors: the characteristics of the magnetic carrier itself, as well as the characteristics of the magnetic field source (including the geometric parameters of permanent magnets, as well as the strength of the magnetic field they generate). Development of a technology for successful magnetically guided delivery of cytostatics should involve development of appropriate magnetic nanoscale drug carriers, assessing their safety, and development of visualisation protocols that allow tracking of their movement in the body.

In this study, we mathematically calculated the optimal magnetic field characteristics for controlling magnetic nanoscale drug carriers in the body. The possibility of retaining MNPs passing through a vessel wall under the influence of an applied magnetic field with these calculated characteristics was also studied in isolated rat vessels. In addition, we synthesised a conjugate of MNPs and a fluorescent agent, and developed a protocol for their visualisation in the body. Under in vivo conditions, in tumour model mice, the efficiency of accumulation of the MNPs in tumour tissue was studied when they were systemically administered under the influence of a magnetic field.

\section{Materials and Methods MNPs}

In the in vitro study, we used reference MNPs, while in the in vivo study, we used MNPs coated with a lactic acid polyester (polylactide, PLA) containing a fluorescent agent (indocyanine; ICG), MNPs-ICG included in the shell, were used (MNP-PLA-EDA-ICG).

\section{MNP Synthesis}

The synthesis of MNPs and their physicochemical characteristics have been described in detail elsewhere. ${ }^{7,8}$

\section{Synthesis of MNPs-ICG}

Synthesis of the PLA-ICG conjugate:

To synthesise the MNPs-ICG, a PLA-ICG conjugate was first produced. A powdered racemic mixture of PLAD and PLA-L, with a molecular weight of $60 \mathrm{kDa}$, was used.

Since both PLA and ICG are acids, in order to synthesise the PLA-ICG conjugate, it was first necessary to synthesise an amino-terminated spacer on the PLA, which facilitated chemisorption of ICG onto the spacer. The spacer was synthesised using ethylenediamine (EDA), using the carbodiimide method and a water-soluble carbodiimide, 1-ethyl-3-(3-dimethylaminopropyl) carbodiimide (EDAC). The PLA-EDA spacer was synthesised as follows. A 20fold molar excess of EDA and a 20 -fold molar excess of EDAC were added to $2 \mathrm{~mL}$ of a $0.1 \mathrm{~g} / \mathrm{mL}$ solution of PLA in chloroform. The synthesis was carried out in a $15-\mathrm{mL}$ polypropylene test tube on a shaker with a revolution of 300 $\min ^{-1}$ for $2 \mathrm{~h}$. The synthesis scheme is illustrated in Figure 1. The synthesis was repeated with a 200 -fold excess of reagents to optimise the synthesis scheme.

At the end of the synthesis, the solution was centrifuged, for 5 minutes at a speed of $3000 \mathrm{~min}^{-1}$, to remove the excess of precipitated polyethylene derivatives. Then, $2 \mathrm{~mL}$ of a solution of ICG in dimethyl sulfoxide (DMSO) 

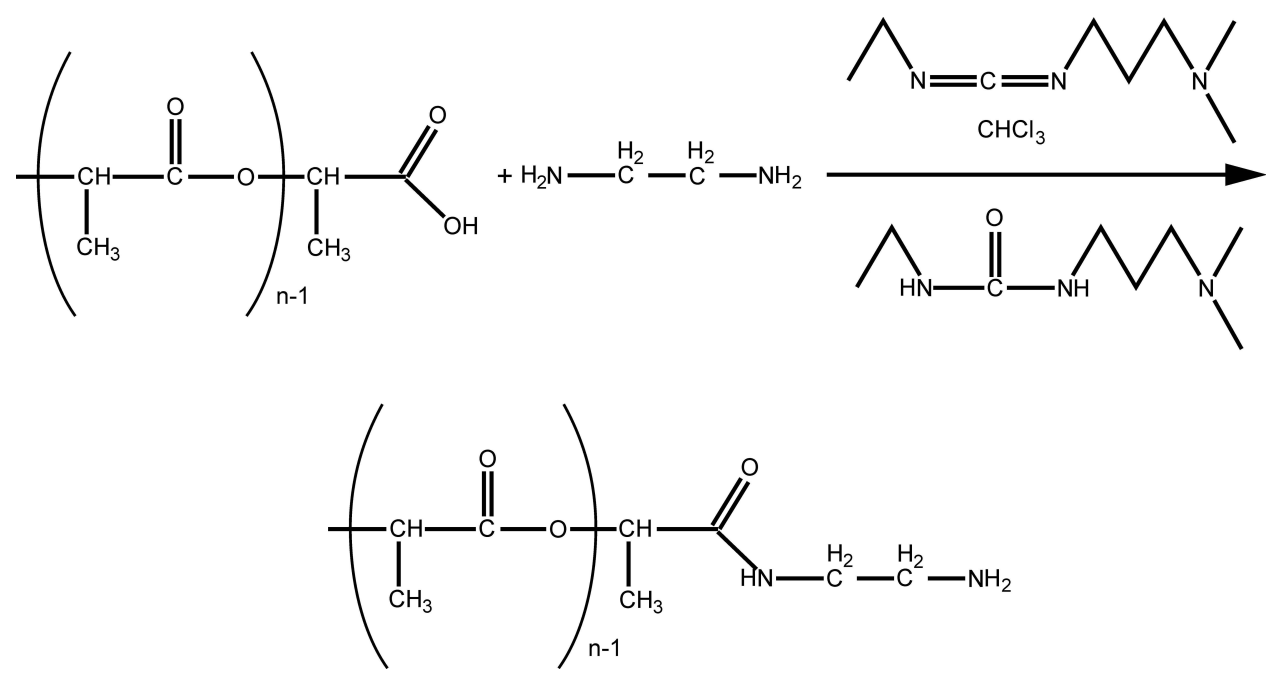

Figure I Scheme of the synthesis of an amino spacer on polylactide.

at a concentration of $0.5 \mathrm{mg} / \mathrm{mL}$ was added to $2 \mathrm{~mL}$ of the solution. Immobilisation was carried out on a shaker for 2 $\mathrm{h}$ at a stirring speed of $300 \mathrm{~min}^{-1}$. A schematic of the resulting conjugate is shown in Figure 2.

Application of the PLA-EDA-ICG conjugate coating to MNPs:

To $200 \mathrm{mg}$ of MNPs, we added $4 \mathrm{~mL}$ of the PLA-EDA -ICG conjugate. This suspension was stirred using an LS220 shaker (LOIP, Russia) for $30 \mathrm{~min}$ at a frequency of $300 \mathrm{~min}^{-1}$. Then, three isopropyl alcohol washes were performed, with magnetic separation. IPA was added to the suspension under constant ultrasonic action for 5-10 min, using a UZD-2 ultrasonic disperser (FSUE NII
TVCH, Russia). After the third IPA wash, the precipitate was washed with distilled water and resuspended in physiological saline at a concentration of $2 \mathrm{mg} / \mathrm{mL}$.

\section{Investigation of the Characteristics of MNPs-ICG}

The size distribution of the obtained MNPs in aqueous solutions was studied using a ZetaSizer Ultra device (Malvern Instruments, UK). The shape and size of the MNPs were studied using transmission electron microscopy (TEM) with a JEM-1400 STEM field emission cathode (JEOL, Japan).

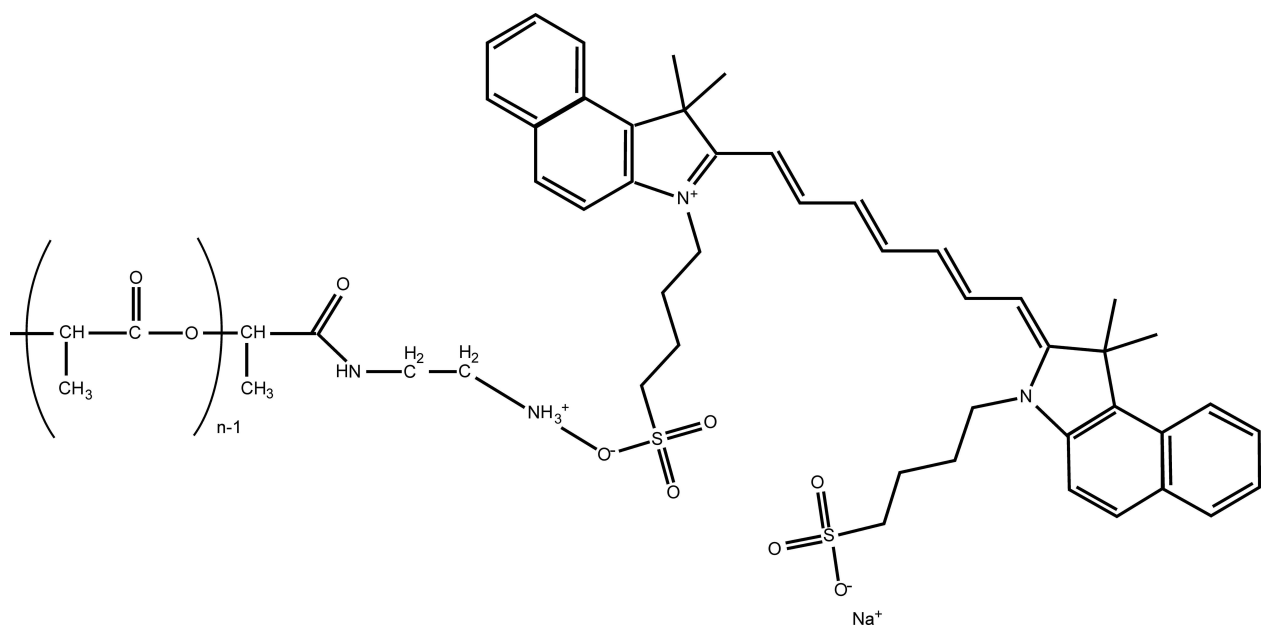

Figure 2 PLA-EDA-ICG conjugate.

Abbreviation: PLA-EDA-ICG, polylactide-ethylenediamine-indocyanine. 


\section{Determination of the Optimal Geometric} Characteristics of Cylindrical Permanent Magnets

In the present study, we used permanent $\mathrm{Nd}-\mathrm{Fe}-\mathrm{B}$ magnets (class N35; with a nickel protective coating) with a cylindrical shape and the following standard sizes (longaxis length $\times$ cylinder diameter): $0.5 \times 2 \mathrm{~mm}, 2 \times 2 \mathrm{~mm}$, $3 \times 2 \mathrm{~mm}$, and $5 \times 2 \mathrm{~mm}$.

\section{Study of the Efficiency of Targeted Transport of MNP in Model Systems \\ in vitro}

The in vitro studies of MNP transport in a model system were carried out on a hydrodynamic stand developed at the Institute of Experimental Medicine of the Almazov National Medical Research Centre of the Ministry of Health of Russia. The volume of the circulating liquid (distilled water or Krebs-Henseleit solution) was $225 \mathrm{~mL}$. Axially magnetised cylindrical magnets were used as the permanent magnets. The magnets were placed on the stand at a distance of $1.5 \mathrm{~mm}$ from the inner wall of a central glass tube, with their ends facing in the direction of the tube (perpendicular). The fluid flow rate inside the closed loop was $60 \mathrm{~L} / \mathrm{h}$ (corresponding to a linear velocity of $0.225 \mathrm{~m} / \mathrm{s}$ ). The Krebs-Henseleit solution was used as circulating fluid, as it is an analogue of blood plasma. The dynamic viscosity coefficient of plasma is $1.1-1.3 \mathrm{mPa} \cdot \mathrm{s}^{9}$ The amount of MNPs adsorbed in a magnetic field was determined spectrophotometrically from the concentration of iron in the circulating liquid after the experiment.

In addition, experimental studies were carried out on a modified hydrodynamic stand, which allows determination of the relative permeability of blood vessels. The main elements of the hydrodynamic stand are shown in Figure 3. The main elements of the hydrodynamic stand are a closed loop that simulates a section of the vascular system of the model, and a storage tank. The movement of the model fluid along the contour with the vascular module was provided by a peristaltic pump. During the experiments, gasification and the required temperature regime were maintained, and the system parameters (temperature, pressure, liquid flow rate, and $\mathrm{pH}$ level) were monitored.

The chamber with the vessels consisted of three containers: one external large and two small containers through which the arms of the central circuit pass. Cannulas were inserted inside the small containers onto

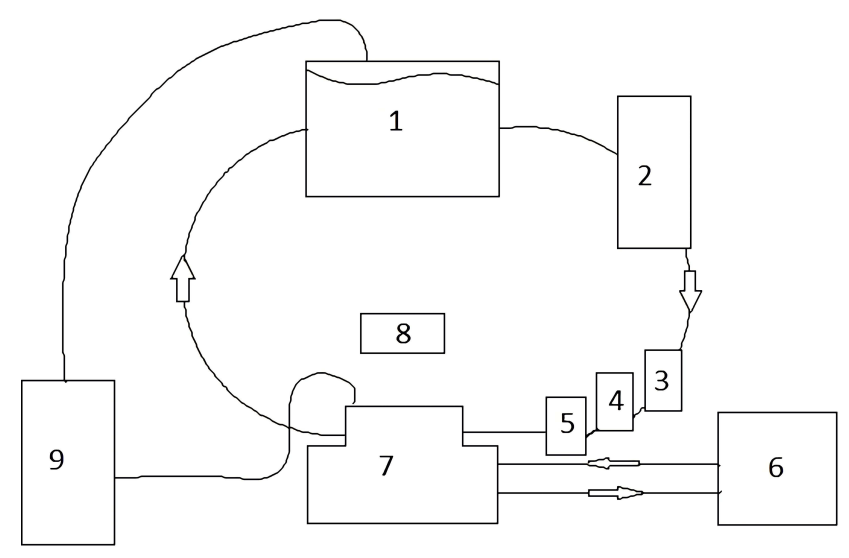

Figure 3 Block diagram of the setup for studies of the permeability of the walls of the carotid artery. I - storage tank, 2 - peristaltic pump, 3 - mechanism for introducing suspension with MNP into the circuit, 4 - flow meter, 5 - pressure sensor in the circuit, 6 - heat exchanger, 7 - chamber with vessels, 8 - magnetic field source, 9 - balloon with carbogen.

which the vessels were strung and tightly tied with threads to the tips of the cannulas. The space between the large and small containers was filled with distilled water, and the temperature was kept constant due to the connection with heat exchangers. The space inside the small containers was filled with Krebs-Henseleit solution to maintain the vitality of the vessel cells. The storage tank also contained Krebs-Henseleit solution. A gas (carbogen) supply system was used to gasify the solution in the storage tank and in the small containers in the chamber containing the vessels (Figure 4).

To determine the index of the relative permeability of the vessel walls, rat carotid arteries were used.

The MNP suspension $(0.5 \mathrm{~mL})$ was introduced into the system, which had the following characteristics: the total internal volume of the storage tank and connecting tubes in the circuit was $20 \mathrm{~mL}$, and the internal volume in each

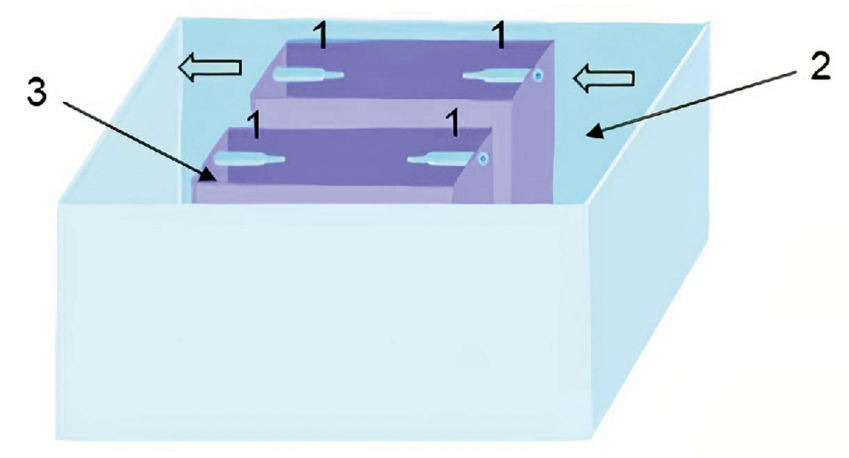

Figure 4 The chamber in which the vessels are placed. I - cannulas for lowering vessels, 2 - external chamber, 3 - small chamber. The arrows show the direction of the model fluid. 
small chamber was $120 \mathrm{~mL}$. The source of the external magnetic field was a permanent magnet, with a standard size of $2 \times 3 \mathrm{~mm}$, which was installed above one of the small chambers at a distance of $1 \mathrm{~cm}$ from the vessel, with its end facing the vessel wall. The temperature was maintained at $37^{\circ} \mathrm{C}$. The power of the roller pump was set to $50 \%$, which corresponded to a speed of $17 \mathrm{~cm} / \mathrm{s}$. As a control, a sample was taken in a small chamber that lacked a permanent magnet.

One hour after the administration of MNPs at a given concentration, fluid samples were taken from the small chambers. The particle concentration was determined spectrophotometrically using an Unico 2802S UV-Vis spectrophotometer (United Products \& Instruments, USA). Measurements were performed at $450 \mathrm{~nm}$, considering the absorption spectra of an MNP suspension.

\section{Murine Models}

All animals were bred and maintained in specific pathogen-free facilities in accordance with the Rus-LASAFELASA guidelines. This study complied with all relevant ethical regulations for animal testing and research and received ethical approval from the Almazov National Medical Research Centre (IACUC). The animals were provided with water ad libitum and were fed regular chow.

The study was carried out on 10 anaesthetized 12-week -old male immunodeficient NSG mice (NOD.CgPrkdescid Il2rgtm1Wjl/Szj, The Jackson Laboratory, USA $)^{10}$, weighing $22 \mathrm{~g} \pm 10 \%$. Immunodeficient mice of this line allow transplantation of human cells and tissues without transplant rejection reactions, due to their suppressed immunity. Littermate animals from different cages were randomly assigned to experimental groups and were either co-housed or systematically exposed to bedding of other groups to ensure equal exposure to common microbiota.

The HeLa human cancer cell line was used to establish a xenograft model. Cells were cultured in DMEM with glutamine (PanEco, Russia) supplemented with $10 \%$ foetal bovine serum (Hyclone, USA), $100 \mathrm{CFU} / \mathrm{mL}$ penicillin, $100 \mu \mathrm{g} / \mathrm{mL}$ streptomycin. The cell line was kindly provided by the Laboratory for Gene Expression Regulation of the Institute of Cytology, Russian Academy of Sciences. Before injection, HeLa cells were removed from the culture plastic with a 1:1 trypsin:Versene solution (Biolot, Russia). After washing, the cells were suspended in complete culture medium to a concentration of $5 \times 10^{6}$ cells per $200 \mu \mathrm{L}$ and diluted (1:1, on ice) with basement membrane matrix (LDEV-FREE, MATRIGEL ${ }^{\circledR}$ CORNING $^{\circledR}$ ). The prepared cell suspension was injected subcutaneously into the skin of the mouse's thigh. Tumour growth was monitored every 3 days using electronic callipers.

A permanent magnet was implanted into the muscle tissue of experimental animals in the immediate vicinity of the tumour when the tumour reached a size of $500 \mathrm{~mm}^{3}$. In the experimental group (MNPs-ICG + tumour-M), $0.1 \mathrm{~mL}$ of MNP suspension was injected, with exposure to a magnetic field. Intact animals with no treatment were used as control (background). Additionally, animals with injection of $0.1 \mathrm{~mL}$ MNPs but without magnet implantation (MNPs-ICG + tumour-BM) were used.

\section{Evaluation of the Fluorescence Intensity of MNPs-ICG in vitro and in vivo}

Fluorescence visualisation of the samples in vivo and in vitro was carried out on an IVIS Lumina LT Series III bioimager (PerkinElmer Inc., USA). For in vitro visualisation, the synthesised PLA-EDA-ICG and MNP-PLA-EDA -ICG conjugates, in a volume of $1 \mathrm{~mL}$, were added to the plate wells. The optimal filters for determining the luminescence intensity of the samples were selected considering the fluorescent properties of the ICG dye: maximum excitation at $745 \mathrm{~nm}$ and emission at $815 \mathrm{~nm}$. The fluorescence intensities of the wells containing conjugates were quantitatively determined using Living Image 4.5.5 software (PerkinElmer Inc.).

Fluorescence intensity and accumulation of MNP-PLA -EDA-ICG conjugates were measured in tumour model mice, in vivo, in the absence and application of a magnetic field at the sites of interest. The mice were anaesthetized with isoflurane anaesthesia, after which they were injected intravenously, via a tail vein, with $0.1 \mathrm{~mL}$ of the MNP-PLA-EDA-ICG conjugate. An untreated mouse was used as a negative control to obtain the fluorescence background. After intravenous administration of conjugates, the animals were placed on a heated stage $\left(37^{\circ} \mathrm{C}\right)$ in the chamber of an IVIS Lumina LT Series III fluorescence imager (PerkinElmer Inc.), while inhalation anaesthesia with $2 \%$ isoflurane was maintained. Signal detection was performed $1 \mathrm{~min}$ after the introduction of the MNPs and after 15 min using the built-in filters for ICG (745$815 \mathrm{~nm})$.

To assess the accumulation of conjugates in the tumour, the peritoneal area of the animals was covered with paper, which made it possible to eliminate the bright fluorescence 
associated with accumulation of the particles in the liver. After studying the biodistribution of MNP-PLA-EDA-ICG, the animals were humanely euthanized by an overdose of isoflurane anaesthesia for subsequent isolation of tumour areas and quantitative assessment of fluorescent radiation. The analysis of signals from the selected regions of interest was processed manually using Living Image 4.5.5 software (PerkinElmer Inc.). Measurements were performed three times for each animal $(n=9)$.

\section{Limitations of This Study}

In this study, we did not quantify the successful loading of ICG on MNPs-ICG. Moreover, we did not compare the retention efficiency of nanoparticles under the influence of permanent magnets of different shapes. Additionally, we did not assess the long-term effect of the magnetic field on the retention of nanoparticles in tumour tissue.

\section{Results}

\section{Characteristics of MNPs-ICG}

The nanoparticles had a dominant fraction with an average size of $195.4 \mathrm{~nm}$. In addition, the suspension contained agglomerates with an average size of $1176.0 \mathrm{~nm}$
(Figure 5A). Subsequently, this fraction was filtered through a centrifugal filter. The zeta potential of the particles was $-15.69 \mathrm{mV}$ (Figure 5B).

The particles were essentially $200 \mathrm{~nm}$ in size (Figure 5C), consisting of individual MNPs, $20 \mathrm{~nm}$ in size, with a less electron-dense organic shell of the PLAEDA-ICG conjugate. The formation of agglomerates in aqueous solutions can be explained by the relatively low modulus of the electrokinetic potential of the individual nanoparticles.

\section{Optimal Geometric Characteristics of Cylindrical Permanent Magnets}

The direction of magnetization of the permanent magnets is shown in Figure 6.

In accordance with the definition of the vector of magnetic induction: ${ }^{11}$

$$
\begin{gathered}
\vec{B}=[\nabla \times \vec{A}], \\
\vec{A}(\vec{x})=\frac{\mu_{0}}{4 \pi} \int^{J_{m}\left(\vec{x}^{\prime}\right)} \mid \overrightarrow{x^{\prime} \mid} d V^{\prime},
\end{gathered}
$$
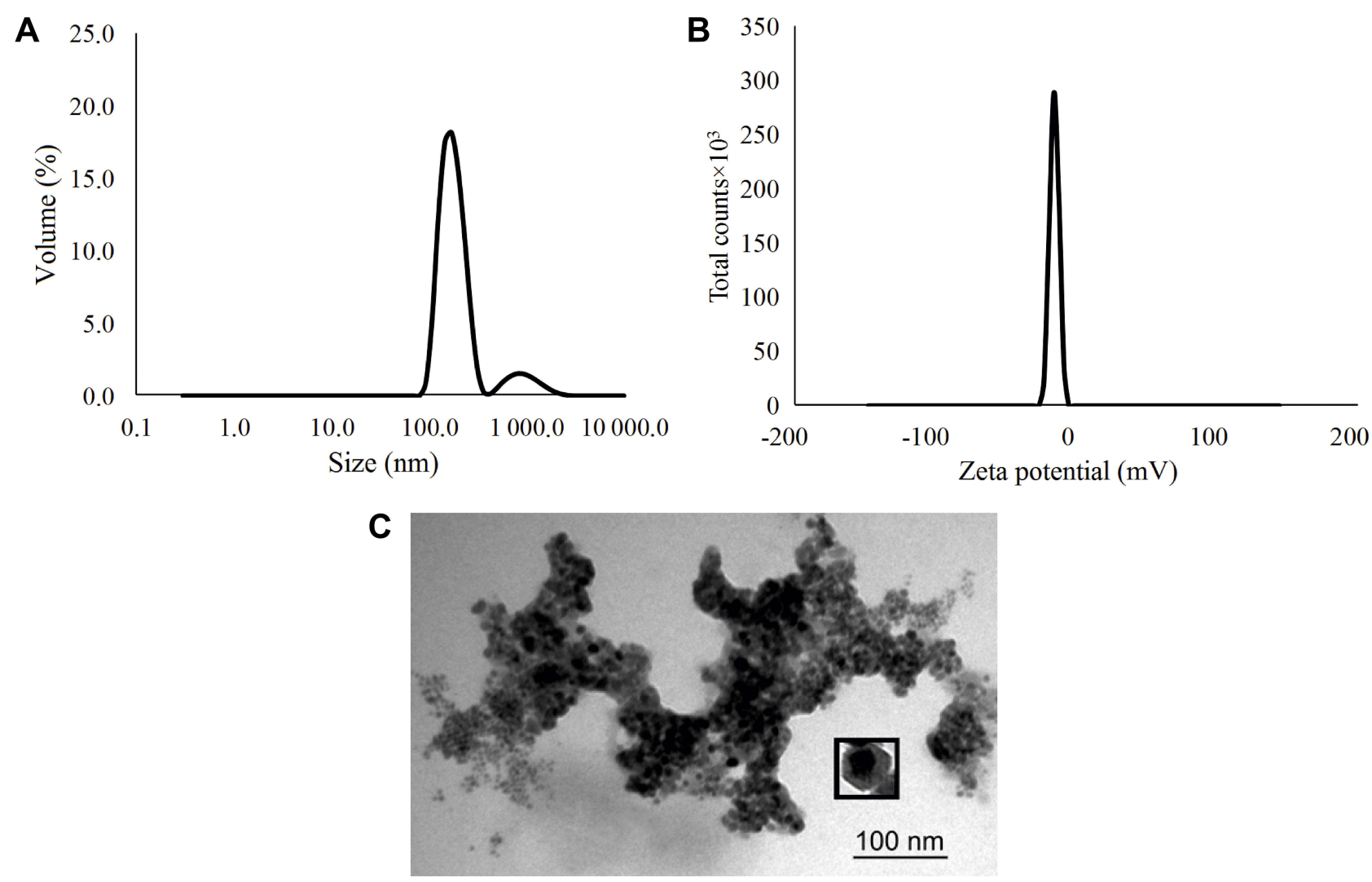

Figure 5 Physical characteristics of the suspension: (A) particle size distribution; (B) distribution of particles over zeta potential; (C) TEM photograph of nanoparticles. 


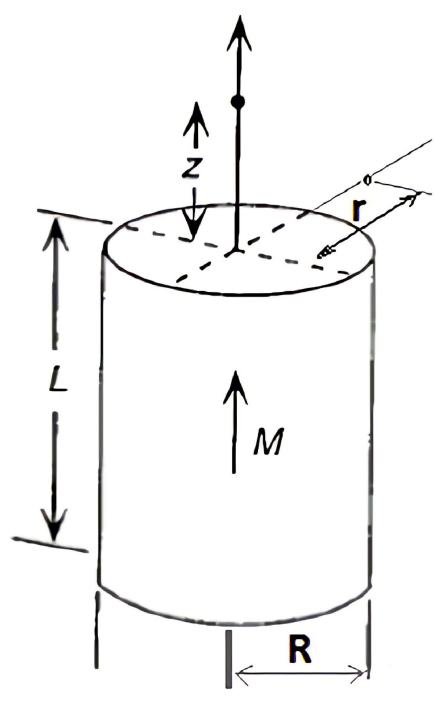

Figure 6 Legend for a permanent magnet.

After substituting the expression for the vector potential, we obtain:

$$
\vec{B}(\vec{x})=\frac{\mu_{0}}{4 \pi} \int\left[\vec{J}_{m}(\vec{x}) \times \frac{\left(\vec{x}-\vec{x}^{\prime}\right)}{\left|\vec{x}-x^{\prime}\right|^{3}}\right] d V^{\prime} .
$$

For a permanent magnet, when the magnetisation is concentrated inside the volume $V$, the integral expression is split into two integrals, namely, over the volume and over the surface:

$$
\begin{aligned}
\vec{B}(\vec{x})= & \frac{\mu_{0}}{4 \pi} \int_{V}\left[\vec{j}_{m}(\vec{x}) \times \frac{\left(\vec{x}-\vec{x}^{\prime}\right)}{\left|\vec{x}-\vec{x}^{\prime}\right|^{3}}\right] d V^{\prime} \\
& +\int_{S}\left[\vec{j}_{m}(\vec{x}) \times \frac{\left(\vec{x}-\vec{x}^{\prime}\right)}{\left|\vec{x}-\vec{x}^{\prime}\right|^{3}}\right] d S^{\prime} .
\end{aligned}
$$

In the case of a sample with constant magnetisation, the current density is zero. Then, the expression for the vector of magnetic induction will take the form:

$\vec{B}(z)=\frac{\mu_{0}}{4 \pi} \int_{-L}^{0} \int_{0}^{2 \pi}\left[M_{S} \vec{\varphi} \times \frac{\left(z \vec{z}-\left(R \vec{r}+\varphi^{\prime} \vec{\varphi}+z^{\prime} \vec{z}\right)\right) R d \varphi^{\prime} d z^{\prime}}{\left[R^{2}+\left(z-z^{\prime}\right)^{2}\right]^{\frac{3}{2}}}\right]$.

The components can be described as:

$$
\begin{gathered}
B_{z}(z)=\frac{\mu_{0}}{4 \pi} \int_{-L}^{0} \int_{0}^{2 \pi} \frac{M_{s} R^{2} d z^{\prime} d \varphi^{\prime}}{\left[\left(z-z^{\prime}\right)^{2}+R^{2}\right]^{\frac{3}{2}}}, \\
B_{z}(r)=\frac{\mu_{0}}{4 \pi} \int_{-L}^{0} \int_{0}^{2 \pi} \frac{M_{s} R(r-R) d z^{\prime} d \varphi^{\prime}}{\left[\left(z-z^{\prime}\right)^{2}+r^{2}+R^{2}-2 r R \cos (\varphi)\right]^{\frac{3}{2}}},
\end{gathered}
$$

$$
B_{r}(z)=\frac{\mu_{0}}{4 \pi} \int_{-L}^{0} \int_{0}^{2 \pi} \frac{M_{s} R z^{\prime} d z^{\prime} d \varphi^{\prime}}{\left[\left(z-z^{\prime}\right)^{2}+r^{2}+R^{2}-2 r R \cos (\varphi)\right]^{\frac{3}{2}}},
$$

Numerical calculations were performed using the MATLAB program (MathWorks, Inc., USA), academic license of ETU "LETI" No. 40502181.

As shown in Figures 7Figures 8Figures 9Figures -10, the strongest field is created by a magnet directed in the axial direction from the end of the cylinder. The effective radius of action is comparable to the geometric dimensions of the magnet. In cylindrical magnets, where the length of the cylinder is greater than its diameter, the strongest field is observed in the axial-radial directions (for the corresponding components); therefore, cylinders with a larger aspect ratio (diameter to length) would be the most effective for MNP adsorption.

\section{Efficiency of Directed Transport of MNPs in Model Systems in vitro}

Special hydrodynamic models can be used to study the methods for MNP delivery to tumour tissue, concentrate nanoparticles in the target area, and determine the behaviour of nanoparticles under the hydrodynamic conditions of the circulatory system. Permanent magnets can be used as external magnetic fields. If we neglect the magnetostatic interaction between nanoparticles, and do not consider the magnetic fluid model, then it is sufficient to estimate the interaction between a magnet and an individual nanoparticle in a dipole-dipole approximation.

Magnetic dipole field (magnetic induction):

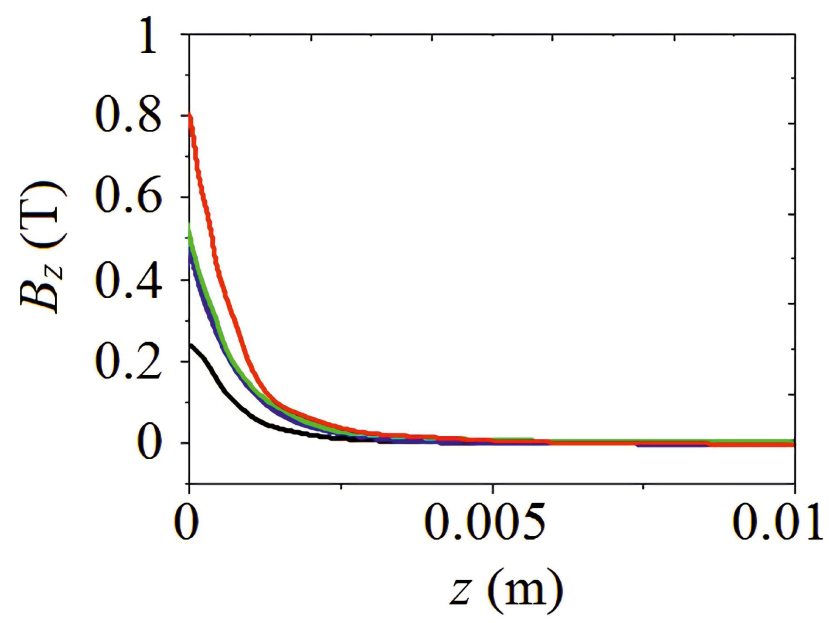

Figure 7 The component of the magnetic induction $B_{z}$ along the axis of the magnet $\mathrm{Oz}$; magnet standard size: black line $0.5 \times 2 \mathrm{~mm}$, blue line $2 \times 2 \mathrm{~mm}$, green line $3 \times 2 \mathrm{~mm}$, red line $5 \times 2 \mathrm{~mm}$. 


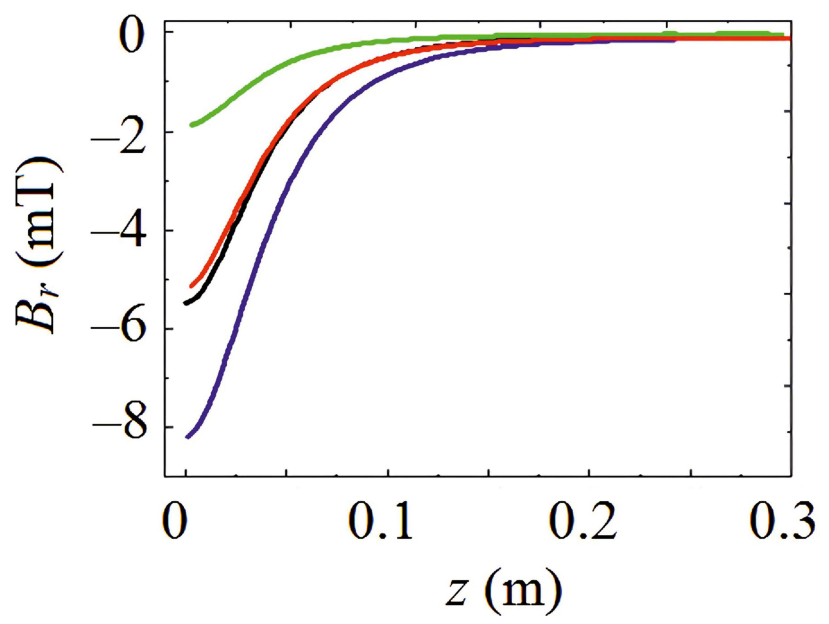

Figure 8 Magnetic induction component $B_{r}$ perpendicular to the magnet axis $\mathrm{Oz}$; magnet standard size: black line $0.5 \times 2 \mathrm{~mm}$, blue line $2 \times 2 \mathrm{~mm}$, green line $3 \times 2 \mathrm{~mm}$, red line $5 \times 2 \mathrm{~mm}$.

$$
\boldsymbol{B}=k\left[\frac{3(\boldsymbol{m}, \boldsymbol{r}) \boldsymbol{r}}{r^{5}}-\frac{\boldsymbol{m}}{r^{3}}\right],
$$

where $\mathbf{m}$ is the magnetic moment of the magnet, $\mathbf{r}$ is the radius vector of the point at which the nanoparticle is located, and $k$ is the system factor. In the dipole approximation, the field of the magnet has a similar configuration (Figure 11).

In a uniform magnetic field, the nanoparticles only rotate along the lines of force. In an inhomogeneous magnetic field, a force acts on it:

$$
F=\left(m, \frac{\partial B}{\partial l}\right)
$$

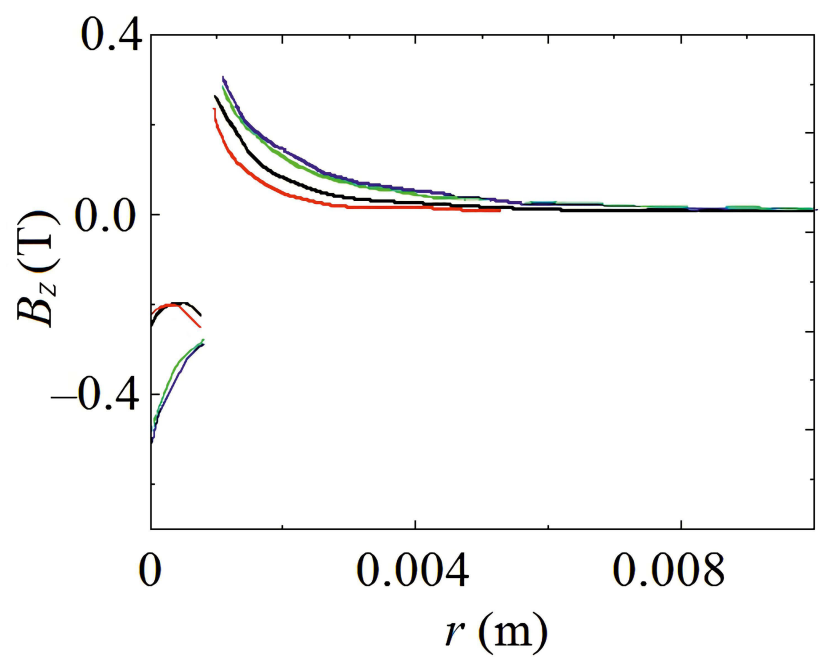

Figure 9 The component of the magnetic induction $B_{z}$ at a distance $r$ from the axis at the end of the magnet $(z=0)$; magnet standard size: black line $0.5 \times 2 \mathrm{~mm}$, blue line $2 \times 2 \mathrm{~mm}$, green line $3 \times 2 \mathrm{~mm}$, red line $5 \times 2 \mathrm{~mm}$.

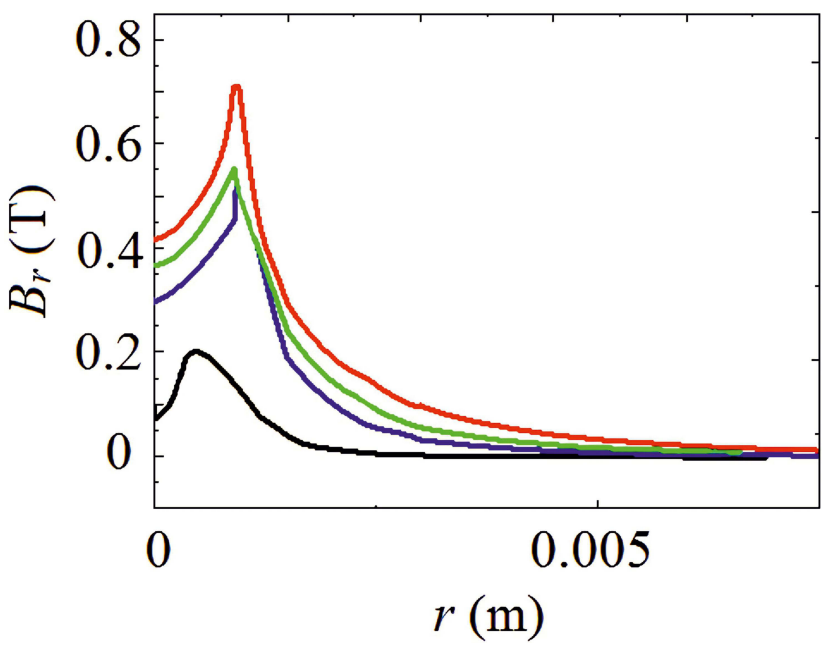

Figure 10 The component of the magnetic induction $B_{r}$ along the radial direction; magnet standard size: black line $0.5 \times 2 \mathrm{~mm}$, blue line $2 \times 2 \mathrm{~mm}$, green line $3 \times 2 \mathrm{~mm}$, red line $5 \times 2 \mathrm{~mm}$.

where $\frac{\partial B}{\partial l}$ is the derivative in a given direction $l$. Moreover, the force pulls the nanoparticle into the region where the field is most inhomogeneous, ie, the curvature and density of the lines of force increase.

Thus, it is desirable to use a sufficiently strong magnet (or a chain of magnets) with pronounced axial anisotropy in the area where the particles are located.

The ability of individual magnets to serve as a sufficient magnetic field source for capturing and retaining MNPs in the vascular bed at the site of application of the field are presented in Table 1.

It follows from the table that, under the given conditions, if distilled water is chosen as the circulating liquid, approximately $50 \%$ of MNPs are sequestered from the flow and retained within $1 \mathrm{~h}$. After $2 \mathrm{~h}$, this percentage increased to more than $90 \%$. On the other hand, if Krebs-Henseleit

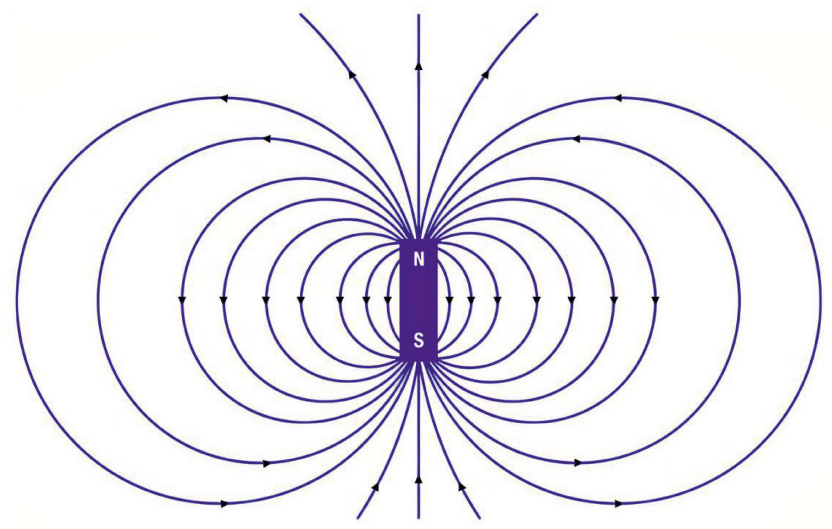

Figure I I Permanent magnet field lines. 
solution is used as model liquid, fewer MNPs are sequestered and retained. For instance, after $2 \mathrm{~h}$, depending on the length of the magnet, only $70-80 \%$ of the MNPs were retained.

The most effective attraction was provided by magnets with a large aspect ratio. The area of action of the magnetic field is comparable to the geometric dimensions of the magnet.

Table 2 presents the results of measurements of the concentration of magnetite MNPs obtained during in vitro tests on isolated vessels.

As shown in Table 2, the use of a permanent magnet to localise MNPs ensured their accumulation in the area of interest. In contrast, in the control group, where no magnetic field was applied, accumulation was not observed. Thus, the use of neodymium magnets allows the selective accumulation of MNPs.

\section{In vitro Study of the Fluorescence of the Obtained Samples}

As can be seen by Sample 1 in Table 3, physical adsorption of ICG to the MNPs is essentially non-existent.
During synthesis, the fluorescence ability of the PLAEDA-ICG conjugate was confirmed to be high. This made it possible to predict the corresponding efficiency of the conjugate as a coating for MNPs. Indeed, we found that MNPs-ICG demonstrated sufficient luminous efficacy to function in fluorescence tomography.

Figure 12 illustrates the measured luminous efficacy of the samples. The pure PLA-EDA-ICG conjugate shows good fluorescent parameters, suitable for measurement on a fluorescence tomograph. When applied to MNPs, a sample with a 200-fold excess of reagents lost fluorescent properties. On the other hand, a sample with a 20 -fold excess of reagents retained fluorescence. Therefore, MNPs-ICG with a 20 -fold excess of reagents was used in the experiment.

Evaluation of the Efficiency of Magnetically Guided Delivery of MNPs-ICG in vivo

The dynamics of the distribution of MNPs-ICG in the bodies of tumour-bearing mice are shown in Figure 13.

Table I Quantitative Determination of Magnetized MNP

\begin{tabular}{|c|c|c|c|c|c|c|}
\hline & $\begin{array}{l}\text { Time, } \\
\text { s }\end{array}$ & $\begin{array}{c}\text { Volume of Injected } \\
\text { MNP, mL }\end{array}$ & $\begin{array}{c}\text { MNP } \\
\text { Concentration, } \mathrm{mg} / \mathrm{mL}\end{array}$ & $\begin{array}{l}\text { Magnet } \\
\text { Size, mm }\end{array}$ & $\begin{array}{l}\text { Liquid Flow } \\
\text { Rate, m/s }\end{array}$ & Liquid Used \\
\hline 1 & 1800 & 5 & I & $3 \times 2$ & 0.3 & $\begin{array}{l}\text { Krebs-Heseleit } \\
\text { solution }\end{array}$ \\
\hline 2 & 7200 & 5 & I & $3 \times 2$ & 0.225 & $\begin{array}{l}\text { Krebs-Heseleit } \\
\text { solution }\end{array}$ \\
\hline 3 & 7200 & 5 & I & $|x|$ & 0.225 & $\begin{array}{l}\text { Krebs-Heseleit } \\
\text { solution }\end{array}$ \\
\hline 4 & 900 & 2 & I & $3 \times 2$ & 0.225 & $\begin{array}{l}\text { Krebs-Heseleit } \\
\text { solution }\end{array}$ \\
\hline 5 & 900 & 2 & I & $3 \times 2$ & 0.225 & Distilled water \\
\hline 6 & 900 & 2 & I & $|x|$ & 0.225 & $\begin{array}{l}\text { Krebs-Heseleit } \\
\text { solution }\end{array}$ \\
\hline 7 & 300 & 2.5 & 2 & $5 \times 2$ & 0.225 & Distilled water \\
\hline 8 & 900 & 2.5 & 2 & $5 \times 2$ & 0.225 & Distilled water \\
\hline 9 & 1800 & 2.5 & 2 & $5 \times 2$ & 0.225 & Distilled water \\
\hline 10 & 3600 & 2.5 & 2 & $5 \times 2$ & 0.225 & Distilled water \\
\hline II & 7200 & 2.5 & 2 & $5 \times 2$ & 0.225 & Distilled water \\
\hline 12 & 7200 & 5 & I & $5 \times 2$ & 0.225 & $\begin{array}{c}\text { Krebs-Heseleit } \\
\text { solution }\end{array}$ \\
\hline
\end{tabular}

Notes: MNP, magnetite nanoparticles. 
Table 2 Measured Values of the Concentration of Magnetite MNP on a Spectrophotometer

\begin{tabular}{|l|c|}
\hline Presence of a Magnetic Field & After I h, $\mathbf{~ m g ~ ( N ~ = ~ 6 ) ~}$ \\
\hline Yes & $0.033 \pm 0.004$ \\
No (Control) & 0 \\
\hline
\end{tabular}

Table 3 Measured Values of Luminous Efficacy of Fluorescent Emission of Samples

\begin{tabular}{|l|c|c|}
\hline $\begin{array}{l}\text { № } \\
\text { Sample }\end{array}$ & $\begin{array}{c}\text { Sample } \\
\text { Composition }\end{array}$ & $\begin{array}{c}\text { Total Luminous Efficiency, } \\
\frac{p / s}{\mu W / \mathrm{cm}^{2}} \times 10^{-8}\end{array}$ \\
\hline 1 & MNP-PLA-ICG & 0.31 \\
2 & PLA-EDA-ICG & 2120.0 \\
3 & MNP-ICG & 42.72 \\
\hline
\end{tabular}

Notes: For an ICG excitation filter at $745 \mathrm{~nm}$, the ICG extraction filter, the luminous efficacy value is indicated by background subtraction.

Abbreviations: MNP-PLA-ICG, magnetite nanoparticles-polylactide-indocyanine; PLA-EDA-ICG, polylactide-ethylenediamine--indocyanine; MNP-ICG, indocyaninemagnetite nanoparticles.

One minute after the administration of MNPs-ICG to tumour model mice in the absence of a magnetic field (group MNPs-ICG + tumour-WM), the MNPs-ICG predominantly accumulated in the liver, with an intensity of $9.884 \times 10^{8} \frac{\mathrm{p} / \mathrm{s}}{\mu \mathrm{W} / \mathrm{cm}^{2}}$, which increased over time, and reached $1.322 \times 10^{9} \frac{\mathrm{p} / \mathrm{s}}{\mu \mathrm{W} / \mathrm{cm}^{2}}$ after $15 \mathrm{~min}$ (Figure $13 \mathrm{~A}$ and $\mathrm{B}$ ). Accumulation of the conjugate in the liver is indicative of the metabolic pathway of these nanoparticles. When tumour model mice were injected with MNPs-ICG and exposed to a magnetic field (group MNPs-ICG + tumour$\mathrm{M})$, the luminescence intensity in the liver was $7.000 \times 10^{8}$ $\frac{p / s}{\mu W / \mathrm{cm}^{2}}$ at $1 \mathrm{~min}$ after injection of MNPs-ICG. The intensity of luminescence in the liver of the animals did not change over time, but it increased within the tumour, indicating active accumulation of MNPs-ICG in the tumour under the influence of a magnetic field.

Figure 14 shows the comparative fluorescence intensity in the tumour $15 \mathrm{~min}$ after the administration of MNPs-ICG to mice. To exclude a liver fluorescence signal arising as secondary radiation from the tumour, the liver area was shielded with white paper. As shown in Figure 14, a significant accumulation of the conjugate, with an intensity of $5.162 \times 10^{8} \frac{\mathrm{p} / \mathrm{s}}{\mu \mathrm{W} / \mathrm{cm}^{2}}$, was observed in the area exposed to a magnetic field. Interestingly, the accumulation of MNPsICG was also observed in animals that were injected with MNPs-ICG, but that were not exposed to a magnetic field (Figure 14A). This suggests passive targeted delivery, which is based on the difference in permeability between healthy blood vessels and tumour vessels. The MNPs-ICG accumulated at the boundary of the permeability zone. To clarify this process, the profile of the average luminous efficacy in a horizontal section was taken (Figure 14C), which confirmed the accumulation of MNPs-ICG in the tumour zone, even in the absence of a magnetic field, and the significantly greater accumulation when a magnet was used. The section
A

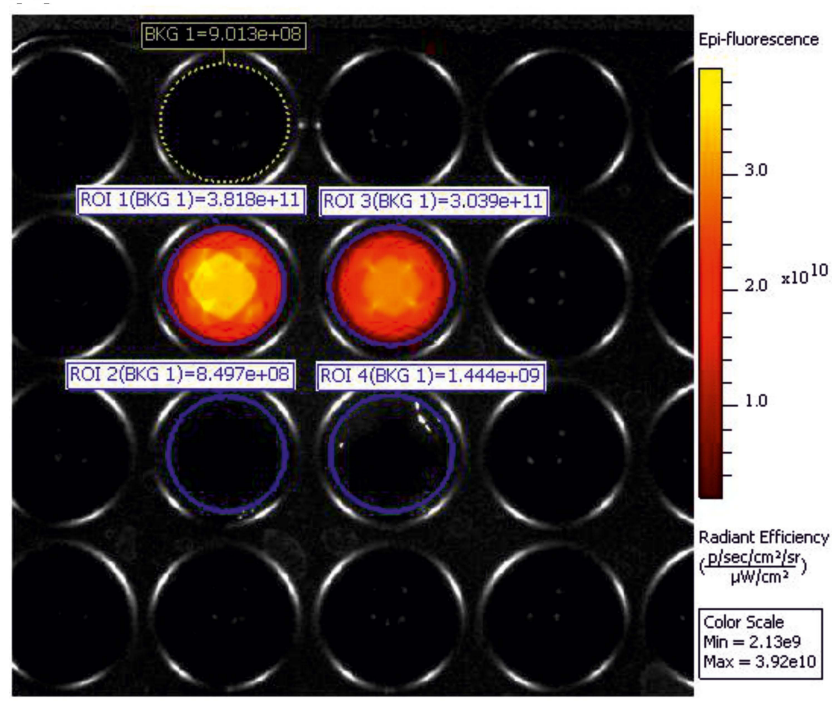

B

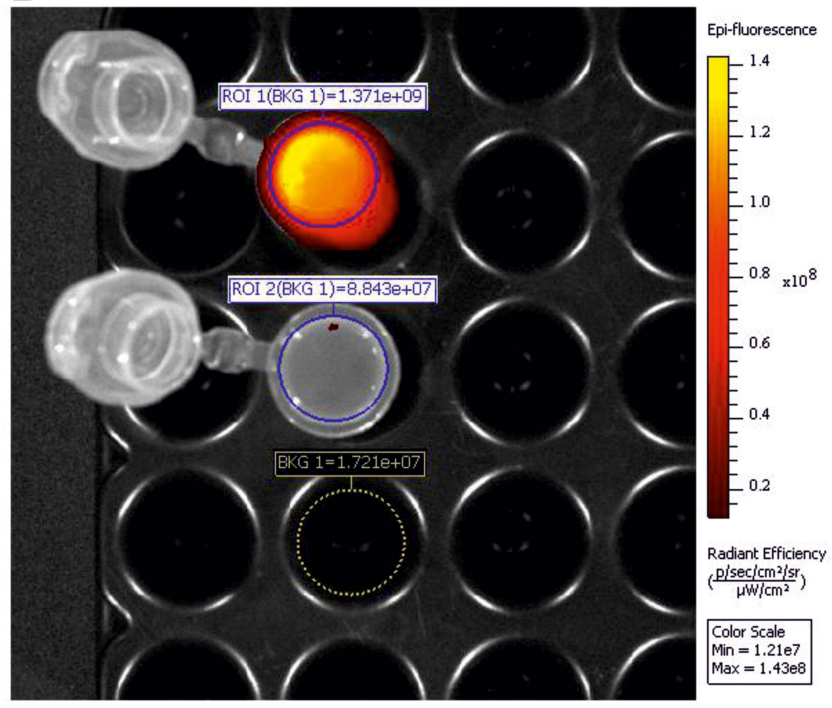

Figure 12 Fluorescence of in vitro samples: (A and B) - from left to right 20-fold excess of reagents; 200-fold excess of reagents (A) PLA-EDA-ICG conjugate; (B) MNPPLA-EDA-ICG.

Abbreviations: MNP-PLA-EDA-ICG, magnetite nanoparticles-polylactide-ethylenediamine-indocyanine; PLA-EDA-ICG, polylactide-ethylenediamine-indocyanine. 
A

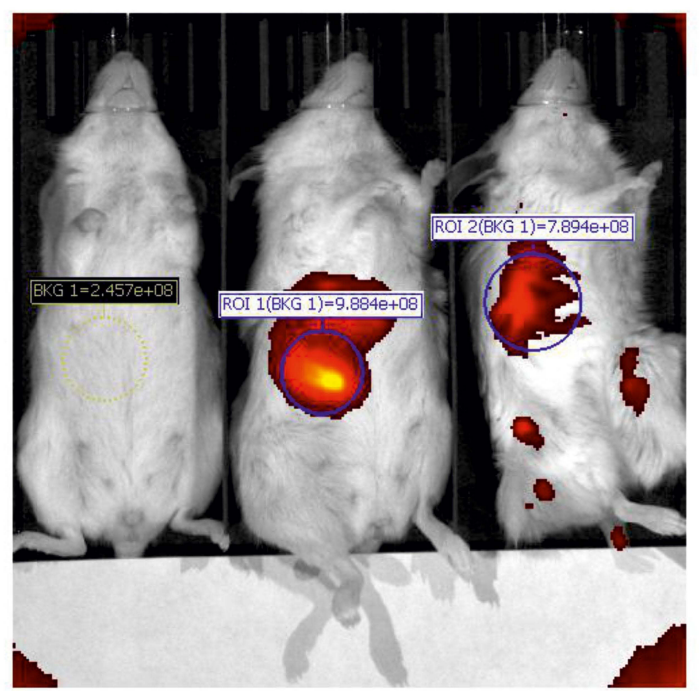

B

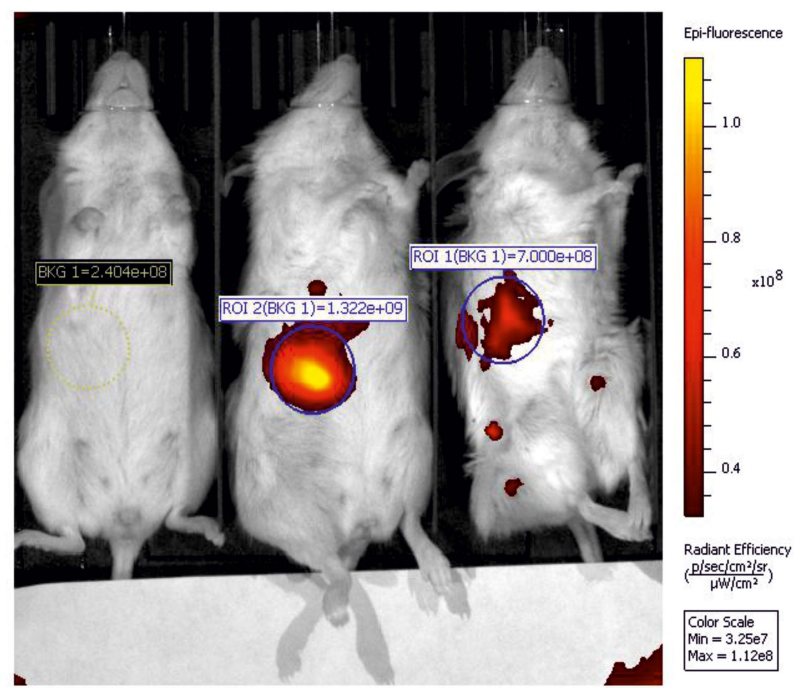

Figure I3 Accumulation of MNPs-ICG in the liver at different points in time: (A and B) - from left to right — control animal; introduction of MNPs-ICG; introduction of MNPs-ICG with the creation of a magnetic field in the area of the tumor; (A) I minute after administration; (B) I5 minutes after administration (arrow indicates location of tumor).

Abbreviation: MNPs-ICG, magnetite nanoparticles-indocyanine.

in which the luminous efficacy profile was measured is shown by the red and green arrows in Figure 14B.

The affected organ was removed from control and experimental mice, and the luminous efficacy was measured. The total luminous efficacy of a tumour removed from an animal that had been injected with MNPs-ICG and exposed to a magnetic field was $1.292 \times 10^{9} \frac{\mathrm{p} / \mathrm{s}}{\mu \mathrm{W} / \mathrm{cm}^{2}}$, as compared to $1080 \times 10^{8} \frac{\mathrm{p} / \mathrm{s}}{\mu \mathrm{W} / \mathrm{cm}^{2}}$ in control animals that were not exposed to a magnetic field (Figure 14D). This trend was consistent with the findings of the in vitro experiment.

Thus, the application of a magnetic field to the tumour site significantly increased accumulation of MNPs in the tumour, but it did not completely eliminate accumulation of MNPs in the liver.

\section{Discussion}

In this study, as part of the development of the concept of magnetically controlled delivery of cytostatic drugs into tumour tissue, MNPs conjugated with a fluorescent agent (ICG, a cyanine dye used in medical diagnostics) that was included in the polylactide coating, was synthesised. To implement the concept itself, it was necessary to ascertain the optimal magnetic characteristics of magnets used as a means of controlling nanoparticles in the body. ${ }^{6}$

It is necessary to know the spatial distribution of the external magnetic field and the vector magnitude of the magnetic force involved in controlling the MNPs. In addition, it is necessary to consider the hydrodynamic characteristics of the particles when they move in a fluid flow. In this study, we calculated the magnetic field of axially magnetised permanent magnets (Nd-Fe-B). Only the area directly near the end of the magnet, at a distance comparable to the linear dimensions of the magnet, is effective. Thus, the largest force is generated closer to the edge of the base of the magnetic cylinder, and the value of the force decreases rapidly with the distance from the magnet. These data are in good agreement with the results obtained by a computer simulation method used in a previous study of the force effect of a high-gradient magnetic field, created by a system of permanent magnets, on the motion of MNPs in the flow of a model fluid. ${ }^{12}$

We proved that, when distilled water is used as the circulating liquid, the MNPs are effectively retained in the application area of magnets with the calculated characteristics in a model on a hydrodynamic stand. The results obtained verified the mathematical calculations used. In order to approximate real clinical conditions better, we performed a separate series of experiments using KrebsHenseleit solution, which is an analogue of blood plasma, as a circulating fluid. An insignificant decrease in the efficiency of accumulation of MNPs in the magnet application area was observed, which is probably due to the change in the interaction of the magnet with MNPs in a medium that has an ionic strength different from that of distilled water. The interaction between MNPs in a solution is generally 
A

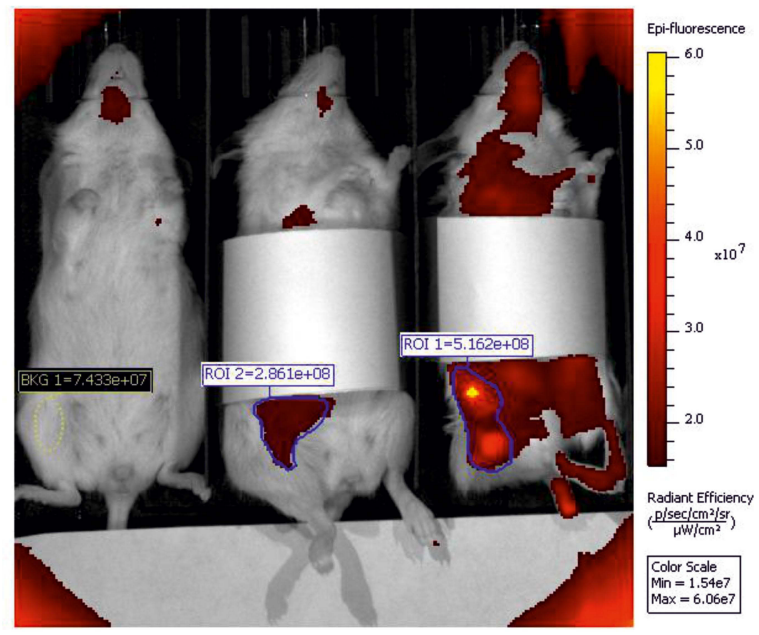

B

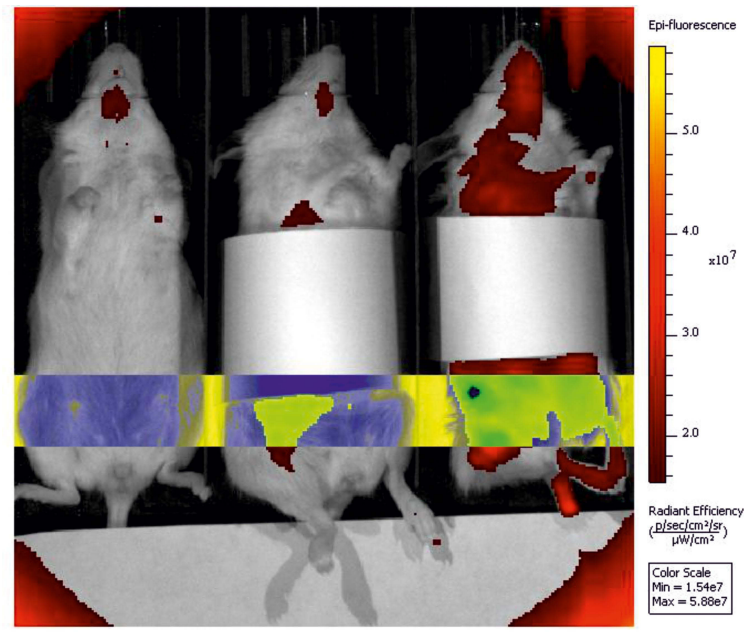

C

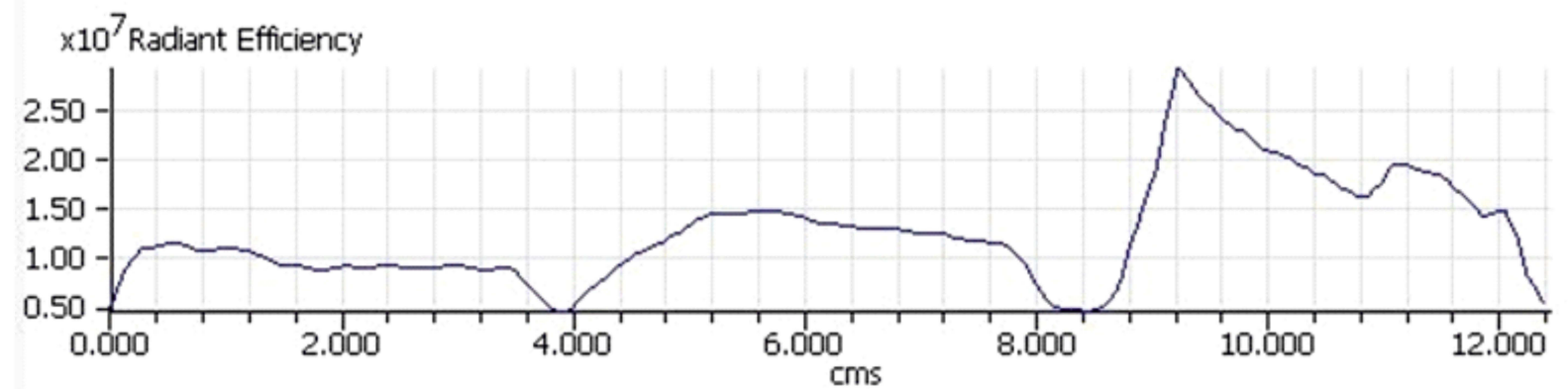

D
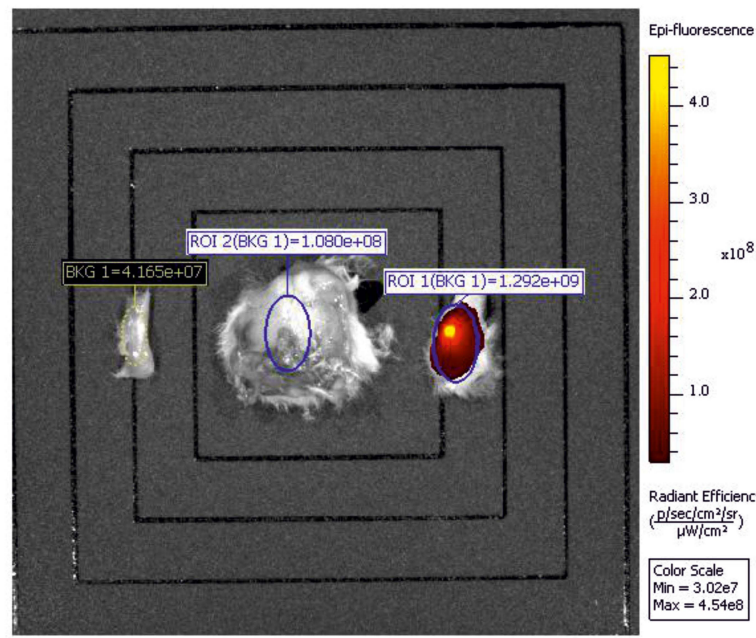

Figure 14 Accumulation of MNPs-ICG in a tumor: (A-C) from left to right - control animal; introduction of MNPs-ICG; introduction of MNPs-ICG with the creation of a magnetic field in the area of the tumor; (A) accumulation of MNPs-ICG in the tumor; (B) cross-section of the horizontal fluorescence measurement map; (C) light output in a horizontal section; (D) fluorescence of the removed organ, from left to right: background (control), organ with the introduction of the conjugate, organ with the introduction of the conjugate and exposure to a magnetic field (arrow indicates location of tumor).

Abbreviation: MNPs-ICG, magnetite nanoparticles-indocyanine.

determined by the balance of the energy of electrostatic repulsion, van der Waals attraction, and dipole-dipole (magnetostatic) interaction. ${ }^{13}$ As was previously shown for MNPs with an inorganic silica shell, ${ }^{8}$ the ionic strength of a solution (changed by varying the sodium chloride content in the range of 0.11-7.2 wt.\%) affects the surface charge of MNPs and, consequently, the electrostatic interaction between them. An increase in ionic strength has a similar effect on MNPs with an organic coating of polyethylenimine and hyaluronic acid, ${ }^{14}$ as well as polyethylene glycol. ${ }^{15}$ The reduced 
influence of the strength of the magnetic field gradient relative to the Coulomb repulsion can therefore lead to a decrease in the efficiency of confinement of MNPs in a magnetic field. Thus, we had proved the possibility of retaining MNPs in a target area by applying an external magnet with specified characteristics.

To confirm the possibility of retaining MNPs passing through the vessel wall under the influence of an applied magnetic field, experiments were performed using a modified hydrodynamic stand, which enabled the assessment of the accumulation of the MNPs passing through the wall of an isolated vessel under the action of a magnetic field. We showed that individual cylindrical Nd-Fe-B permanent magnets can serve as a sufficient external magnetic field source to ensure the passage of MNPs through the walls of isolated vessels, providing the necessary retention and accumulation in the target area. These results are consistent with previous similar studies. ${ }^{16,17}$ The results obtained make it possible to consider the use of such MNPs as a means of targeted drug delivery.

We investigated the distribution of MNPs-ICG in tumour model mice to confirm the concept of magnetically controlled delivery of MNPs to the site of the tumour. In these experiments, we used implanted magnets with the described characteristics as the source of the magnetic field. We showed that MNPs actively accumulated in the area where the tumour was located; however, implementation of a magnetic field at the tumour site did not completely eliminate the accumulation of MNPs in the liver. The passive accumulation of MNPs, primarily in the liver, represents a challenge for modern nanomedicine. We also observed a maximum MNPs-ICG fluorescence signal in the liver, even when the presence of a magnetic field directed the MNPs-ICG to the tumour growing in the animal's thigh. The enhanced permeability and retention effect allows MNPs to penetrate and remain in the tumour tissue, but fenestration in the liver blood vessels and other liver cell properties linked to the physiological role of this organ leads to the side effect of preferential MNP accumulation in the liver after intravenous injection of MNPs. ${ }^{18}$ Use of a magnetic field substantially increased accumulation of MNPs in the tumour, but it could not significantly reduce the accumulation of MNPs in the liver.

Several strategies have been proposed to overcome the problem of the preferential biodistribution of nanoparticles in the liver. Active targeting of MNPs with the help of tumour-specific ligands will make the association of nanoparticles with tumour cells stronger and more specific.
Prevention of macrophage uptake by MNPs, including uptake by resident liver phagocytes and Kupffer cells, can be achieved by several methods, including PEGylation of nanoparticles. Densely PEGylated nanoparticles have an increased blood half-life. ${ }^{19}$

Another possible approach to improve the "cargo" delivery to the tumour site is to load hematopoietic cells with MNPs. Recently, a team from the Harbin Institute of Technology loaded neutrophils with paclitaxel-MNPs embedded in a bacterial membrane. Such "neutrobots" effectively pass the blood-brain barrier and accumulated at a glioma site. In addition to chemokine-driven tumour infiltration by MNP-loaded neutrophils, the implementation of a rotating magnetic field potentiated the effect and directed neutrophils, with accelerated infiltration speed. ${ }^{20}$

To conclude, we believe that using a magnetic field in combination with the aforementioned approaches is a viable strategy to improve nanoparticle-targeted delivery and to shift the balance from liver accumulation to intratumoral infiltration. The results obtained suggested that the type of MNPs used in combination with the calculated magnetic field can form the basis for the development of a means of magnetically controlled transport of cytostatic drugs into tumour tissue. MNP functionalization by an erythrocyte membrane may also be considered as a promising approach to tumour targeting. ${ }^{21}$ It can be assumed that the combination of the nano- and cellular approaches will allow avoidance of excessive accumulation of MNPs in the liver and will improve the efficiency of targeted delivery to tumour sites.

\section{Conclusion}

In magnets, where the length of the cylinder is greater than its diameter, the strongest field is observed in the axial-radial directions; therefore, elongated cylinders should be used as the most effective for sequestering MNPs. The most effective attraction of MNPs is provided by elongated cylindrical magnets (where the length is greater than the diameter). In this case, the area of action of the magnetic field was comparable to the geometric dimensions of the magnet. Separate cylindrical permanent neodymium magnets can serve as a sufficient external magnetic field source to capture magnetite nanoparticles from model vessels in a hydrodynamic stand, and causing accumulation of nanoparticles in the target area. Using in vitro experiments with a vascular module for retaining MNPs under the influence of a magnetic field, we showed that the use of a permanent magnet ensures accumulation of MNPs in the region of interest. In tumour model mice, with exposure to a constant magnetic field, the 
accumulation of MNPs increased in the area where the tumour was located. Thus, MNPs, based on iron oxide, can act as a promising means for targeted delivery of cytostatics. The data obtained can form the basis for the development of magnetically controlled transport of cytostatics.

\section{Acknowledgments}

The authors are grateful to Nikita V Zhilyakov for help in creating figures for the review and Peter $\mathrm{V}$ Kharitonskii for mathematical modeling.

\section{Funding}

The work on animal models was financially supported by the Ministry of Science and Higher Education of the Russian Federation (Agreement No. 075-15-2020-901).

\section{Disclosure}

The authors report no conflicts of interest in this work.

\section{References}

1. Bray F, Ferlay J, Soerjomataram I, Siegel RL, Torre LA, Jemal A. Global cancer statistics 2018: GLOBOCAN estimates of incidence and mortality worldwide for 36 cancers in 185 countries. CA Cancer J Clin. 2018;68(6):394-424. doi:10.3322/caac.21492

2. Pucci C, Martinelli C, Ciofani G. Innovative approaches for cancer treatment: current perspectives and new challenges. Ecancermedicalscience. 2019;13:1-26. doi:10.3332/ecancer.2019.961

3. Zhou Q, Wei Y. For better or worse, iron overload by Superparamagnetic iron oxide nanoparticles as a MRI contrast agent for chronic liver diseases. Chem Res Toxicol. 2017;30(1):73-80. doi:10.1021/acs.chemrestox.6b00298

4. Chang D, Lim M, Goos JACM, et al. Biologically targeted magnetic hyperthermia: potential and limitations. Front Pharmacol. 2018;9 (8);831. doi:10.3389/fphar.2018.00831

5. Liu XL, Choo ESG, Ahmed AS, et al. Magnetic nanoparticle-loaded polymer nanospheres as magnetic hyperthermia agents. $J$ Mater Chem B. 2014;2(1):120-128. doi:10.1039/c3tb21146k

6. Subramanian M, Miaskowski A, Jenkins SI, Lim J, Dobson J. Remote manipulation of magnetic nanoparticles using magnetic field gradient to promote cancer cell death. Appl Phys a Mater Sci Process. 2019;125 (4):1. doi:10.1007/s00339-019-2510-3

7. Toropova $\mathrm{YG}$, Golovkin AS, Malashicheva $\mathrm{AB}$, et al. In vitro toxicity of $\mathrm{Fe}_{\mathrm{m}} \mathrm{O}_{\mathrm{n}}, \mathrm{Fe}_{\mathrm{m}} \mathrm{O}_{\mathrm{n}}-\mathrm{SiO}_{2}$ composite, and $\mathrm{SiO}_{2}-\mathrm{Fe}_{\mathrm{m}} \mathrm{O}_{\mathrm{n}}$ core-shell magnetic nanoparticles. Int $J$ Nanomedicine. 2017;12:593-603. doi:10.2147/IJN.S122580

International Journal of Nanomedicine

\section{Publish your work in this journal}

The International Journal of Nanomedicine is an international, peerreviewed journal focusing on the application of nanotechnology in diagnostics, therapeutics, and drug delivery systems throughout the biomedical field. This journal is indexed on PubMed Central, MedLine, CAS, SciSearch ${ }^{\mathbb{B}}$, Current Contents ${ }^{\mathbb{B}} /$ Clinical Medicine, $^{2}$
8. Vezo OS, Gareev KG, Korolev DV, et al. Aggregate stability and magnetic characteristics of colloidal $\mathrm{Fe}_{\mathrm{m}} \mathrm{O}_{\mathrm{n}}-\mathrm{SiO}_{2}$ particles obtained by sol-gel method. Phys Solid State. 2017;59(5):1008-1013. doi:10.1134/S1063783417050304

9. Késmárky G, Kenyeres P, Rábai M, Tóth K. Plasma viscosity: a forgotten variable. Clin Hemorheol Microcirc. 2008;39(14):243-246. doi:10.3233/CH-2008-1088

10. Shultz LD, Lyons BL, Burzenski LM, et al. Human lymphoid and myeloid cell development in NOD/LtSz- scid IL2R $\gamma$ null mice engrafted with mobilized human hemopoietic stem cells. J Immunol. 2005;174(10):6477-6489. doi:10.4049/ jimmunol.174.10.6477

11. Landau LD, Lifshitz EM. The classical theory of fields. In: Course of Theoretical Physics. 4th ed. Vol. 2; 1975. Pergamon Press, Oxford, UK. 89-108.

12. Kirilenko A, Chekhun B, Podoltsev A, et al. Analysis of the force impact of a high gradient magnetic field on magnetic nanoparticles in a fluid stream. Rep Natl Acad Sci Ukraine. 2010;324(8):162-172.

13. Shliomis MI. Magnetic fluids blums.pdf. Sov Phys - Uspekhi. 1974;17(2):153-169. doi:10.1070/PU1974v017n02ABEH004332

14. Al-Deen FN, Selomulya C, Williams T. On designing stable magnetic vectors as carriers for malaria DNA vaccine. Colloids Surf B. 2013;102:492-503. doi:10.1016/j.colsurfb.2012.09.026

15. Park YC, Smith JB, Pham T, et al. Effect of PEG molecular weight on stability, T2 contrast, cytotoxicity, and cellular uptake of superparamagnetic iron oxide nanoparticles (SPIONs). Colloids Surf B. 2014;119:106-114. doi:10.1016/j.colsurfb.2014.04.027

16. Seliger C, Jurgons R, Wiekhorst F, et al. In vitro investigation of the behavior of magnetic particles by circulating artery model. $J$ Magn Magn Mater. 2007;311(1):358-362. doi:10.1016/j. jmmm.2006.10.1205

17. Heidsieck A, Vosen S, Zimmermann K, Wenzel D, Gleich B. Analysis of trajectories for targeting of magnetic nanoparticles in blood vessels. Mol Pharm. 2012;9(7):2029-2038. doi:10.1021/ mp3001155

18. Van Haute D, Berlin JM. Challenges in realizing selectivity for nanoparticle biodistribution and clearance: lessons from gold nanoparticles. Ther Deliv. 2016;8(9):763-774. doi:10.4155/tde2017-0057

19. Perrault SD, Walkey C, Jennings T, Fischer HC, Chan WCW. Mediating tumor targeting efficiency of nanoparticles through design. Nano Lett. 2009;9(5):1909-1915. doi:10.1021/n1900031y

20. Zhang H, Li Z, Gao C, et al. Dual-responsive biohybrid neutrobots for active target delivery. Sci Robot. 2021;6(52):eaaz9519. doi:10.1126/scirobotics.aaz9519

21. Xia Q, Zhang Y, Li Z, Hou X, Feng N. Red blood cell membrane-camouflaged nanoparticles: a novel drug delivery system for antitumor application. Acta Pharm Sin B. 2019;9(4):675-689. doi:10.1016/j.apsb.2019.01.011
Journal Citation Reports/Science Edition, EMBase, Scopus and the Elsevier Bibliographic databases. The manuscript management system is completely online and includes a very quick and fair peer-review system, which is all easy to use. Visit http://www.dovepress.com/ testimonials.php to read real quotes from published authors. 